OPEN ACCESS

Edited by: Carlos Roncero,

University of Salamanca Health

Care Complex (Complejo

Asistencial Universitario de

Salamanca/CAUSA), Spain;

University of Salamanca, Spain

Reviewed by:

Lara Grau-López,

Hospital Universitari Vall

d'Hebron, Spain

Giuseppe Carrà,

Università degli studi di

Milano Bicocca, Italy

*Correspondence:

Jorge Gaete

jgaete@uandes.cl

Specialty section:

This article was submitted to Addictive Disorders, a section of the journal Frontiers in Psychiatry

Received: 15 September 2017

Accepted: 22 January 2018

Published: 08 February 2018

Citation:

Gaete J, Rojas G, Fritsch R and Araya $R$ (2018) Association between School Membership and Substance Use among Adolescents.

Front. Psychiatry 9:25

doi: 10.3389/fpsyt.2018.00025

\section{Association between School Membership and Substance Use among Adolescents}

\author{
Jorge Gaete ${ }^{1,2 *}$, Graciela Rojas ${ }^{3,4}$, Rosemarie Fritsch $^{3}$ and Ricardo Araya ${ }^{5}$ \\ ${ }^{1}$ Department of Public Health and Epidemiology, Faculty of Medicine, Universidad de los Andes, Santiago, Chile, ${ }^{2}$ Centre for \\ Global Mental Health, Department of Population Health, London School of Hygiene and Tropical Medicine, London, United \\ Kingdom, ${ }^{3}$ Departamento de Psiquiatría y Salud Mental, Clínica Psiquiátrica Universitaria, Universidad de Chile, Santiago, \\ Chile, ${ }^{4}$ Millennium Institute for Research in Depression and Personality, Santiago, Chile, ${ }^{5}$ Health Service and Population \\ Research Department, Institute of Psychiatry, Psychology and Neuroscience, King's College London, London, \\ United Kingdom
}

Background: Substance use among adolescents is a major problem worldwide, producing many health and economic consequences. Even though there are well-known personal, familial, and social factors associated with drug use, less is known about the effect of school-related factors. School membership is a recognized variable affecting academic performance among students; however, its effect on substance use is less understood.

Aims: The primary aim of this study was to explore the association between school membership and cigarette, alcohol, and cannabis use among a representative sample of secondary students from municipal state-funded schools in Santiago of Chile, and secondly, to test the hypothesis that depressive or anxiety symptoms mediate this association.

Methods: A total of 2,508 students from 22 state-funded schools in Santiago, Chile, answered a questionnaire. This instrument included an abbreviated version of the psychological sense of school membership (PSSM), questions regarding the use of alcohol, tobacco, and cannabis and scales of psychological functioning (depression, anxiety, self-concept, and problem-solving). The association analyses were performed using adjusted regression models for each outcome using all independent variables while controlling for gender and age. For the mediation effect, a combination of ordinary least square and logistic regression analyses was conducted.

Results: There was an association between a strong PSSM and low risk for smoking (OR 0.57; 95\% Cl 0.46-0.72), drinking (0.65; 95\% Cl: 0.51-0.83), and cannabis use (0.52; 95\% Cl 0.37-0.74). We also found that depressive and anxiety symptoms do not fully mediate the association between school membership and any substance use, and $73 \%$ of this effect in the case of smoking, $80 \%$ in the case of drinking, and $78.5 \%$ in the case of cannabis use, was direct.

Conclusion: This is the first study in Latin America exploring the association between school membership and substance use among secondary students. School membership 
seems to be an important and independent factor to be included in preventive interventions. Therefore, these results support future research aiming to test interventions at increasing the sense of school membership to prevent substance use among adolescents.

\section{Clinical Trial Registration: ISRCTN19466209. Retrospectively registered.}

Keywords: school membership, substance abuse, adolescents, depression, anxiety, mental health

\section{INTRODUCTION}

Substance use among adolescents is still a major problem worldwide producing many health and economic consequences (1). The most recent Chilean governmental report about substance use among 8th-12th graders says that the monthly prevalence of smoking, drinking, and cannabis use was $26.7,35$, and $18.8 \%$, respectively. There has been no change in smoking and drinking behavior from the previous study, and there has been a clear increase in cannabis use over the last 10 years. These figures are very high when compared with other parts of the world. For example, in European secondary students, among all counties studied, it was found that an average of $21 \%$ of adolescents have used cigarettes; $48 \%$, have used alcohol; and 7\%, have used marihuana in the last 30 days (2). Comparing Chile with the rest of American countries, a recent report found that Chile has the highest prevalence of tobacco use in the last month and the prevalence of cannabis use in the last year of the continent (3) among secondary students. The same report shows that Chile is one of the 14 out of 29 countries studied in America, with a prevalence of alcohol use in the last month over $30 \%$. However, when we observed the rate of progression between students in Year 8 to Year 12, Chile has the third leading place in Latin America (after Ecuador and Peru) increasing three times the prevalence during secondary school years (3).

Although several studies have explored the influence of personal, familial, school peer, and environmental factors on the use of substances of abuse among adolescents (4-6); the effect of school membership seems less well investigated. School membership is a concept of students' perceptions about whether they feel accepted, respected, included, and supported by others in the school social environment (7). It is also defined as the perception of social bonds with other school members; something that will have implications in terms of self-identity and commitment among students of a specific school. It is recognized as an important determinant of school attendance, good behavior, effort in schoolwork (8), and having better academic results (9). The sense of school membership influences the way students become interested and engaged in everyday school activities $(10,11)$. It is also recognized as an important factor in school retention and participation of vulnerable students in their schools (12).

Abbreviations: PSSM, psychological sense of school membership scale; BDI-II, Beck Depressive Inventory, second version; RCADS, revised child anxiety and depression scale; CATS, children's automatic thoughts scale; SPSI-R, social problem-solving inventory revised.
Few studies have investigated the association between school membership (e.g., feeling part of the school, feeling close to, and respected by the people at school) and substance use, and they have been conducted mostly in developed countries $(6,12-19)$. It seems that students who feel connected and part of their school are less likely to be engaged in smoking, heavy drinking, and cannabis use. On the contrary, students who feel less supported and respected by teachers and peers and less part of the school, may feel isolated, depressed or anxious resulting in a higher risk of using drugs, and to failing in school (12). Additionally, students who do not fell part of their schools are less likely to perceive substance use as a problem that may affect their future academic goals (16).

There are potential explanations for the association between school membership and substance use. As Fletcher et al. proposed (20), students who feel disengaged with school might use drugs as a way of bonding with other students who are also disconnected from the schools. Another reason (20) may be that the school environment may be perceived and felt too challenging (from school performance to group acceptance), and difficulties to perform well in this environment might generate frustration and emotional symptoms. Students might resort to using drugs to alleviate some of these symptoms or escape from the school pressure. To our knowledge, no previous studies from Latin America have explored this association and the potential mediation effect of depressive or anxiety symptoms.

The main purpose of this study is to explore the association between school membership and cigarette, alcohol, and cannabis use among a representative sample of secondary students from municipal state-funded schools in Santiago, Chile. A secondary purpose is to test the hypothesis that depressive or anxiety symptoms mediate this association.

\section{MATERIALS AND METHODS}

\section{Setting and Participants}

The data presented here comes from the baseline assessment of the first large cluster randomized controlled trial in Latin America of a universal school-based aimed at reducing depressive symptoms among 9th graders from 22 municipal mixed-sex schools in Santiago, Chile. Informed consent was obtained from parents or main caregivers and assent from the students. All students at Year 9 were eligible to participate in the trial and all but two students provided data for this study. In this original trial, we found no evidence of the proposed intervention being better than usual care in any of the main outcomes. Further information 
about this trial can be found the publications of the protocol (21) and main results (22).

\section{Survey Administration}

After obtaining authorization from the schools and consent from the parents or main caregivers, the evaluation research team approached all selected schools and classes and asked students for their assent. In April 2009, the baseline assessment was carried out using self-administered questionnaires, which took one standard academic hour ( $45 \mathrm{~min}$ ) to be completed. The research team was well trained and they followed a strict protocol with actions to be implemented before (e.g., time and procedure to contact schools to arrange the date of assessment), during (e.g., how to answer questions from students), and after (e.g., how to enter data in pre-built datasets) the study. Teachers were asked to stay in the classroom to help if needed but were encouraged to stay away from pupils' desks while students answered the questionnaires.

\section{Measures}

All scales used in the questionnaire were pre-tested in four schools with similar background the academic year before the baseline assessment to make sure students could understand the questions and respond with consistency $(21,22)$.

\section{Dependent Variables}

We assessed three outcomes regarding substance use: smoking, drinking, and cannabis use. We used standard questions implemented in several school-based surveys internationally (23) and nationally (24) to allow comparisons. We asked for any use of cigarettes, drinking alcohol or smoking cannabis during the last 30 days prior to the survey (dichotomized into 0 "never" versus 1 "at least once").

\section{Independent Variables School Membership}

School membership was assessed using the eight-item abbreviated version of psychological sense of school membership (PSSM) (7), which has been used in other studies $(25,26)$. This scale measures if students feel valued, respected, and included in the school. Each statement may be answered using a scale from 1 "Not at all true" to 5 "Completely true." The score is calculated summing up the answers for each statement and dividing by 8 . A single latent factor was identified after performing an exploratory factor analysis, and the Cronbach alpha for this scale was 0.78 . For the association analysis, we categorized students into tertiles: low (students with the lowest PSSM scores), medium (students in the middle third), and high (students with the highest PSSM scores) school membership. For the analysis of mediation effect, we use this score as a continuous variable.

\section{Other Independent Variables}

Gender and age.

Depression was assessed using the 21-item Beck Depressive Inventory, second version (BDI-II). This is a self-administered scale generating a total score from 0 to 63 . It has been previously validated among Chilean adolescents (27). We dichotomized scores according to our own study of validation using the cut-off of 19/20 for girls and 13/14 for boys (28) to decide if the student was depressed. The Cronbach alpha for this scale was 0.89 .

Anxiety was assessed using the Spanish version of the revised child anxiety and depression scale, but we included just the generalized anxiety, social anxiety, and panic sub-scales because depression was already assessed by the BDI-II and the separation anxiety subscale was less important for this age group. We also dichotomized around the median with a score $\geq 19$ defined as high anxiety. The Cronbach alpha for this scale was 0.83 .

Personal failure subscale of the children's automatic thoughts scalewas used as a measure of self-concept. This 10-item scale gives a good idea of how well (or poorly) students think about themselves and detecting negative thinking (29). We dichotomized around the median with a score $\geq 8$ defined as poor selfconcept. The Cronbach alpha for this scale was 0.88 .

Problem-solving skills were assessed using a modified version of the short version of the social problem-solving inventory revised. This scale assessed several aspects of problem-solving processes. Only 20 out of 25 items were used in this study because students had some problems with the understanding of some items. We dichotomized around the median with a score $\geq 45$ defined as good problem-solving skill. The Cronbach alpha for this scale was 0.90 .

\section{Ethics Approval and Consent to Participate}

This study was approved by the local committee of ethics at the Hospital Clínico Universidad de Chile (No. 179; June 30, 2008), and informed and written consent from parents or main caregivers and informed and written assent from students were required to participate.

\section{Statistical Analysis}

We performed a complete case analysis. First, we examined univariable associations between each independent variable and each outcome using logistic regressions. Then we performed adjusted regression models for each outcome using all independent variables, while also controlling for gender and age.

To explore the mediation effects, we followed guidance on mediation analysis with dichotomous data (30). In view that we had dichotomous dependent variables (smoking, drinking, or cannabis use), continuous independent variables (sense of school membership score), and binary variables as possibly mediating the effect on outcomes, we opted to use a combination of ordinary least square and logistic regression analyses to determine the indirect effect.

All analyses were carried out using STATA version 13.0.

\section{RESULTS}

\section{Descriptive Statistics}

A total of 2,508 students participated in this study. Missing data for the baseline was small (9.4\%). The mean age was 14.5 years old (range: $12-189$ and $44.5 \%$ corresponded to girls).

A $38.7 \%$ of students smoked cigarettes, a $27.5 \%$ drunk alcohol, and a $13.8 \%$ used cannabis in the last 30 days. 
The mean of school membership score was $3.7(\mathrm{SD}=0.7)$.

Almost one in three students had a BDI-II score over the cut-off to be considered depressed. A high score of anxiety $(\geq 19)$ was presented in $53.5 \%$ of students and also, more than half of students had poor self-concept. A $52.5 \%$ had a score under the category of good problem-solving (see Table 1).

\section{Unadjusted Analysis}

In Table 2, we can see that a higher sense of school membership is associated with a lower proportion of consumption of all drugs during the last month. When we observe the unadjusted results, it is possible to see the protective association of school membership: the higher the score the lower the likelihood of being a smoker, drinker, or marijuana user.

\section{Adjusted Analysis}

Students who have a higher level of school membership have a lower likelihood of being smokers (OR 0.57; 95\% CI 0.46-0.72). We can notice that is a trend in this protective effect. Other factors associated with being a smoker were age, being girl, having more depressive symptoms, and poor self-concept (see Table 3 ).

Regarding drinking, we also found a strong reduction in the probability of drinking in the last month among those students who had a high sense of school membership (OR 0.65; 95\% CI

TABLE 1 | Descriptive characteristics, overall and by gender.

\begin{tabular}{lc}
\hline & Overall \\
\cline { 2 - 2 } Variable & $\boldsymbol{n}=\mathbf{2 , 5 0 8}$ \\
\hline Age (12-18), mean (SD) & $14.53(0.89)$ \\
Smoking (last 30 days) & $970(38.74)$ \\
Drinking (last 30 days) & $687(27.47)$ \\
Binge drinking (last 30 days) & $482(19.29)$ \\
Cannabis use (last 30 days) & $345(13.80)$ \\
School Membership, mean (SD) & $3.67(0.72)$ \\
Low-school membership, mean (SD) & $2.90(0.43)$ \\
Medium school membership, mean (SD) & $3.75(0.18)$ \\
High-school membership, mean (SD) & $4.49(0.29)$ \\
BDI-II, mean (SD) & $13.43(10.24)$ \\
Depressed ( $\geq 20$ in girls; $\geq 14$ in boys) & $777(30.98)$ \\
RCADS, mean (SD) & $19.69(8.54)$ \\
High anxiety ( $\geq 19)$ & $1302(53.54)$ \\
Self-concept, mean (SD) & $10.09(7.91)$ \\
Poor self-concept ( $\geq 8)$ & $1,351(54.76)$ \\
Problem-solving skill, mean (SD) & $44.82(12.82)$ \\
Good problem-solving skill ( $\geq 45)$ & $1,269(52.46)$ \\
\hline
\end{tabular}

TABLE 2 | Distribution of substance use behavior according to school membership.

\begin{tabular}{lccc}
\hline $\begin{array}{l}\text { School membership } \\
\text { level }\end{array}$ & $\begin{array}{c}\text { Smoking, } \\
\boldsymbol{n}=\mathbf{2 , 4 7 1}\end{array}$ & $\begin{array}{c}\text { Drinking, } \\
\boldsymbol{n}=\mathbf{2 , 4 6 8}\end{array}$ & $\begin{array}{c}\text { Cannabis } \\
\text { use, } \boldsymbol{n}=\mathbf{2 , 4 6 9}\end{array}$ \\
\hline Low & $\boldsymbol{n}(\mathbf{\%})$ & $\boldsymbol{n}(\mathbf{\%})$ & $\boldsymbol{n}(\mathbf{\%})$ \\
Medium & $419(47.89)$ & $308(35.16)$ & $160(18.29)$ \\
High & $309(37.14)$ & $208(25.06)$ & $\begin{array}{c}113(13.60) \\
64(8.39)\end{array}$ \\
\hline$\chi^{2}(\mathrm{df}), p$ & $227(29.71)$ & $163(21.39)$ & $\begin{array}{c}33.8809(2), \\
p=0.000\end{array}$
\end{tabular}

0.51-0.83). Other variables associated with drinking were age, being depressed and having a poor self-concept (see Table 3).

Finally, a high level of school membership was also associated with lower risk of using cannabis during the last month (OR 0.52; 95\% CI 0.37-0.74). Age, being girl, and poor self-concept were also associated with cannabis use during the last month (see Table 3).

Exploring the mediation effect, we found that being depressed and having a poor self-concept mediated significantly the effect of school membership on smoking (Figure 1). Approximately a $27 \%$ of the effect on school membership is mediated by being depressed and having a poor self-concept. However, a $73 \%$ of the total effect was direct and significant.

For drinking behavior (Figure 2), being depressed mediated part of the effect of school membership on drinking. This mediation reached a significance of up to $20 \%$ of the effect and approximately $80 \%$ was direct and significant.

The association between school membership and using cannabis during the last month was significantly mediated by having a poor self-concept (21.5\%) (Figure 3 ), and $78.5 \%$ of the variance of the association between school membership and cannabis use was direct and significant.

\section{DISCUSSION}

The use of cigarettes, alcohol, and cannabis during the last month is highly prevalent among Chilean adolescents. When comparing our prevalence results with those from others studies we have to be cautious because substance use is highly determined by age. This is because substance use increases gradually as adolescents get older and different countries have different laws regulating the legal age to purchase cigarettes, drink alcohol or to purchase/use cannabis, which ultimately seems to influence adolescent behavior (31). The mean age of our sample was 14.5 years old and results from a recent national survey in New Zealand among a similar population found that $11.4 \%$ of students smoked cigarettes and $10.1 \%$ smoked cannabis in the last month (32). Prevalence for use of all drugs was much lower than in Chile. The Monitoring the Future surveys in the United States include students from 8th, 10 th, and 12th grades making it less comparable with our study. However, in 2014, among 8th and 10th graders, the prevalence of smoking cigarettes, alcohol use, and cannabis use in the last month ranged between 4 and 7.2\%, 9 and 23.5\%, and 6.5 and $16.6 \%$, respectively (33). Therefore, smoking and drinking among ninth grade Chilean students is higher than in American students in 10th grade. Additionally, the trends in cannabis use among Chilean students are changing rapidly, increasing in prevalence at the highest rate since the start of data collection in Chile (24). The last national report of substance use in English adolescents between 14 and 15 years old showed that 21 and 40\%, respectively, had their last alcoholic drink in the last month; while $5-10 \%$ of students 14 and 15 years old, respectively, took cannabis in the last month (34). Taking all of this into account, our results show that substance use in Chile is an urgent problem and resources should be given to better understand the causes of the problem and to the implementation of effective measures to reduce its use.

Depression and anxiety have been found associated with low-academic performance and high-school dropout among 
TABLE 3 | Unadjusted and adjusted associations of predictor variables with smoking, drinking, and cannabis use among Chilean adolescents.

\begin{tabular}{|c|c|c|c|c|c|c|}
\hline \multirow[b]{3}{*}{ Variable } & \multicolumn{2}{|c|}{ Smoking } & \multicolumn{2}{|c|}{ Drinking } & \multicolumn{2}{|c|}{ Cannabis use } \\
\hline & Unadjusted & Adjusted & Unadjusted & Adjusted & Unadjusted & Adjusted \\
\hline & OR $(95 \% \mathrm{Cl})$ & OR $(95 \% \mathrm{Cl})$ & OR $(95 \% \mathrm{Cl})$ & OR $(95 \% \mathrm{Cl})$ & OR $(95 \% \mathrm{Cl})$ & OR $(95 \% \mathrm{Cl})$ \\
\hline \multicolumn{7}{|l|}{ School membership } \\
\hline Low & 1 & 1 & 1 & 1 & 1 & 1 \\
\hline Medium & $0.64(0.53-0.78)^{\star *}$ & $0.73(0.59-0.91)^{*}$ & $0.62(0.50-0.76)^{\star \star}$ & $0.72(0.57-0.90)^{\star}$ & $0.70(0.54-0.91)^{\star}$ & 0.77 (0.57-1.02) \\
\hline High & $0.46(0.38-0.56)^{\star \star}$ & $0.57(0.46-0.72)^{\star *}$ & $0.50(0.40-0.63)^{\star \star}$ & $0.65(0.51-0.83)^{\star \star}$ & $0.41(0.30-0.56)^{\star \star}$ & $0.52(0.37-0.74)^{\star \star}$ \\
\hline Age & $1.86(1.69-2.05)^{\star *}$ & $1.84(1.66-2.05)^{\star \star}$ & $1.66(1.50-1.83)^{\star \star}$ & $1.57(1.42-1.75)^{\star *}$ & $2.06(1.81-2.34)^{* *}$ & $2.00(1.75-2.29)^{\star \star}$ \\
\hline \multicolumn{7}{|l|}{ Gender } \\
\hline Boys & 1 & 1 & 1 & 1 & 1 & 1 \\
\hline Girls & $1.48(1.26-1.74)^{\star \star}$ & $1.46(1.21-1.76)^{\star \star}$ & $1.02(0.86-1.22)$ & 1.00 (0.83-1.22) & $1.30(1.04-1.63)^{\star}$ & $1.33(1.02-1.72)^{\star}$ \\
\hline Depressed & $2.11(1.78-2.51)^{\star *}$ & $1.52(1.22-1.89)^{\star \star}$ & $1.82(1.52-2.20)^{\star \star}$ & $1.43(1.13-1.79)^{\star}$ & $1.99(1.58-2.51)^{\star \star}$ & $1.29(0.97-1.72)$ \\
\hline High anxiety & $1.30(1.10-1.53)^{*}$ & $0.87(0.71-1.06)$ & $1.11(0.93-1.33)$ & $0.84(0.68-1.04)$ & $1.22(0.96-1.53)$ & $0.91(0.69-1.21)$ \\
\hline Poor self-concept & $1.97(1.67-2.33)^{\star *}$ & $1.47(1.19-1.841)^{\star \star}$ & $1.56(1.30-1.87)^{\star \star}$ & $1.28(0.02-1.60)^{\star}$ & $1.86(1.46-2.37)$ & $1.43(1.06-1.93)^{\star}$ \\
\hline Good problem-solving skill & $0.79(0.67-0.93)^{*}$ & $1.00(0.83-1.21)$ & $0.87(0.73-1.04)$ & $1.08(0.89-1.32)$ & $0.68(0.54-0.85)^{\star}$ & $0.84(0.65-1.09)$ \\
\hline
\end{tabular}

${ }^{*} p \leq 0.05$.

${ }^{* *} p \leq 0.001$.

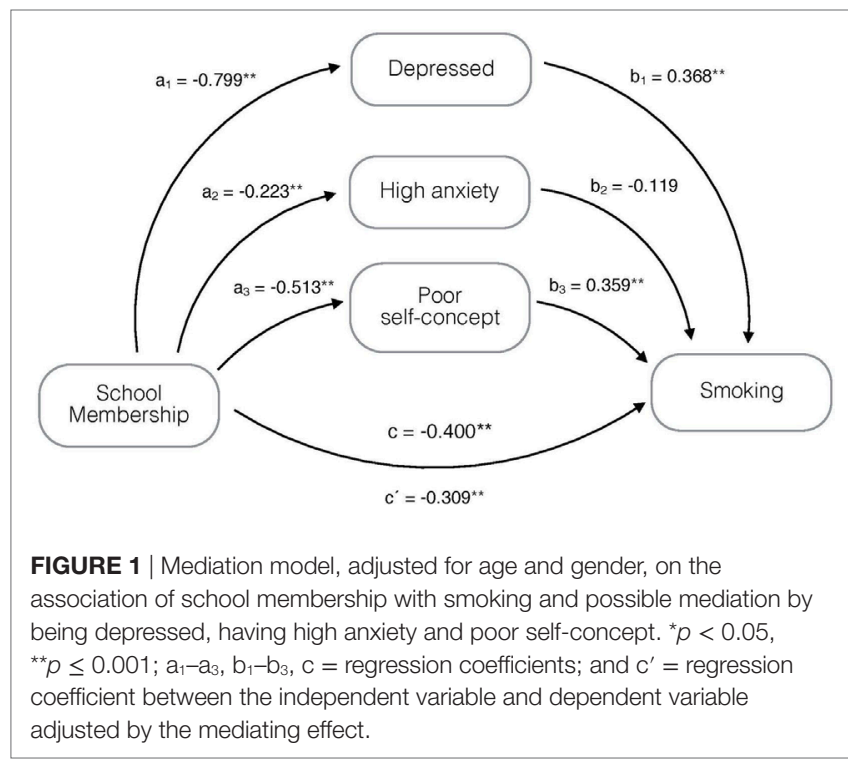

adolescents (35), indicating that students with psychological distress may be less motivated and involved with their schools. Additionally, adolescents with anxiety and depressive symptoms are more likely to be involved in drug use. There is a clear association between depression and anxiety and substance use among adolescents, but it seems to go in both directions (36-39). Some studies show that depressive symptoms are present before the experience of use of alcohol and drugs $(40,41)$, and others show that alcohol and drug use are risk factors for depression (42-44). On the other hand, students with low-school connectedness also have a higher risk for substance use $(45,46)$.

This study adds evidence to the role of anxiety and depressive symptoms in the association between school membership and substance use among adolescents. We found that school membership appears to have a large impact on reducing the risks of substance use behaviors, even after controlling for important individual confounders such as age, gender, or depressive symptoms. Therefore,

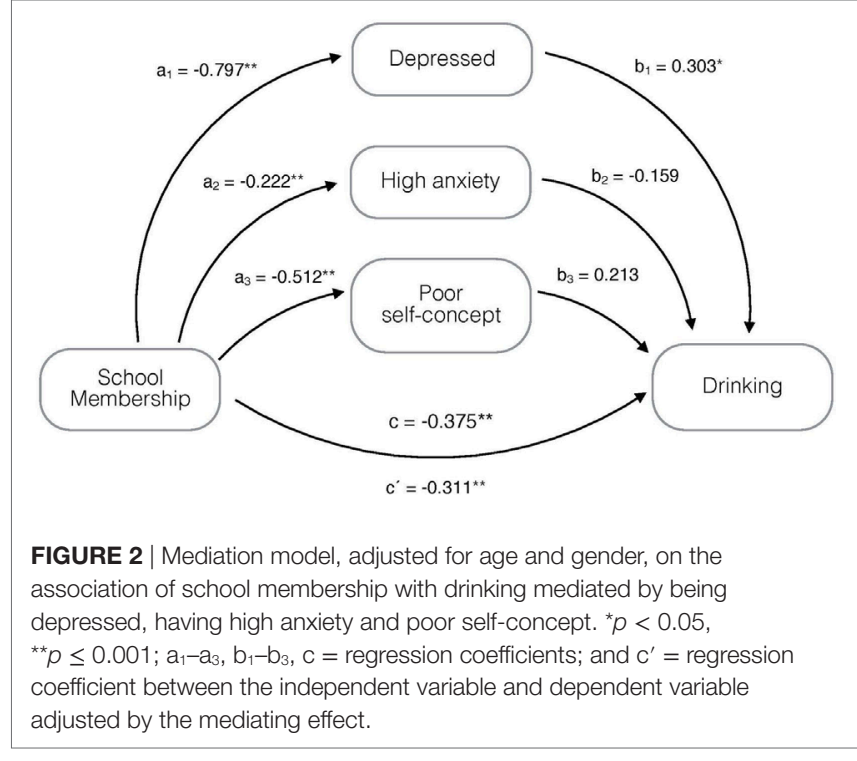

school membership had an independent effect on drug use that seems to not be mediated by personal negative feelings such as depression or poor self-concept. School membership, or other related concepts such as connectedness, attachment, engagement, or bonding, has been considered an important factor influencing academic performance among students. In recent years, studies have related it to mental health and substance use. For instance, Bond et al., using a longitudinal design, found that those students with low-school connectedness at Year 8 had increased risk of anxiety/depressive symptoms, regular smoking, drinking, and use of cannabis in later years (12). However, this research used a multidimensional scale exploring school connectedness, with few items related to sense of school belonging. School connectedness/ bonding should be studied taking into account the independent effect of each dimension. One example of this attempt is our recent study, where we explored the independent association of school commitment and school attachment with smoking, and 


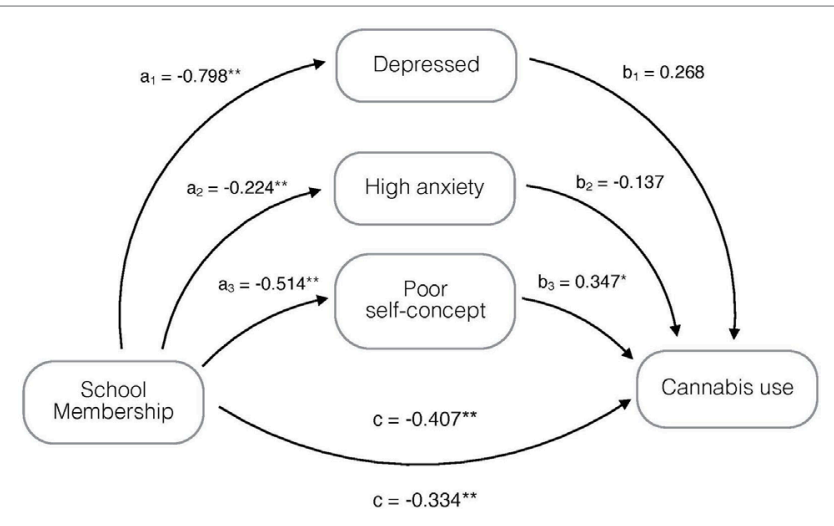

FIGURE 3 | Mediation model, adjusted for age and gender, on the association of school membership with cannabis use mediated by being depressed, having high anxiety and poor self-concept. * $p<0.05$, ${ }^{* *} p \leq 0.001 ; a_{1}-a_{3}, b_{1}-b_{3}, c=$ regression coefficients; and $c^{\prime}=$ regression coefficient between the independent variable and dependent variable adjusted by the mediating effect.

we found that students who committed to their school activities were less likely to smoke (47). However, school attachment, the emotional bonds to the school, was not associated with smoking risk. In the present study, we have only explored the association between sense of school membership and substance use.

Most of the effect of school membership over smoking, drinking, and cannabis use is direct. However, we found some evidence that part of the effect was due to being depressed for the case of smoking and drinking. Additionally, having a poor self-concept accounted for part of the effect for smoking and was the only factor accounting for cannabis use. Mediator variables are not the same for different substances. However, we could think that students disconnected from their schools have some negative feelings (being depressed and poor self-concept) that, partially, may influence their use of different substances. We still need to study what other factors are mediating the effect of school membership and how this association and mediating effects change over time using longitudinal study designs.

We think that our findings support the idea of using schoolbased interventions that promote a sense of school belonging to prevent substance use (45). For instance, some studies have highlighted the benefit of using programs that help to develop constructive commitment and sense of belonging to families, schools, and communities. This is performed through the development of social skills and competences that allow them to communicate effectively, make better decisions, build better self-esteem, and have a greater sense of personal responsibility $(48,49)$. Additionally, interventions that promote positive relationships between teachers and students and a sense of belonging may also help to promote other healthy behaviors (50).

This study had some limitations. It is a cross-sectional study; therefore, we cannot rule out reverse causality in the associations we found. A longitudinal design may help to understand the relation in time between school membership and substance use (46). There may be other potential confounders that were not measured, such as academic performance or peer influence, that may explain some residual confounds. Additionally, all data were collected using self-reported questionnaires, which may have some reporting bias. Although this is a large and representative sample of secondary students attending state founded schools in Santiago of Chile, it may not represent younger or older students in those schools nor students attending subsidized and private schools in Santiago or other cities in Chile. Finally, the 30-day usage of cigarette, alcohol, and cannabis as outcome measures may have limitations to fully understand the experience of substance use among adolescents; however, this is a recommended time interval used in school surveys (23), and the dichotomous approach is widely used, allowing us to compare our findings with other studies $(51,52)$.

\section{CONCLUSION}

This is the first study in Latin America exploring the association between school membership and substance use among secondary students. We found that those students having a high sense of school membership had a lower risk of smoking, drinking, and cannabis use compared with students who had low-school membership. We also found that part of this effect is mediated by the presence of depressive symptoms and poor self-concept. These results provide support to test interventions aimed at increasing the sense of membership to prevent substance use among adolescents.

\section{AVAILABILITY OF DATA AND MATERIALS}

The data can be accessed from the corresponding author through the following address jgaete@uandes.cl, after all main results generated from these data, according to the Funding, are published. The data will be accessed for research purpose and this is because during the ethical clearance process we agree with the Ethical Committee of University of Chile to keep the confidentiality of the data set.

\section{ETHICS STATEMENT}

This study was approved by the local committee of ethics at the Hospital Clínico Universidad de Chile (No. 179; June 30, 2008), and informed consent from parents or main caregivers and assent from students were required to participate.

\section{AUTHOR CONTRIBUTIONS}

RA and JG conceived and designed the study. JG, RF, and GR supervised the collection of data. JG and RA analyzed and interpreted the data, and produced the drafting of the manuscripts. All authors provided a critical revision of the manuscript.

\section{ACKNOWLEDGMENTS}

We also want to thank all of the students and their parents who consented to participation in this study and all of the school authorities who permitted the access to these students. Finally, we want to thank all of the research assistants and data collectors who contributed to this work. 


\section{FUNDING}

We acknowledge the Wellcome Trust for its support of this research (project number $082584 \mathrm{Z} / 07 / \mathrm{Z}$ ). The writing of the manuscript was led by JG while doing a Postdoctoral Research

\section{REFERENCES}

1. Gutierrez A, Sher L. Alcohol and drug use among adolescents: an educational overview. Int J Adolesc Med Health (2015) 27(2):207-12. doi:10.1515/ ijamh-2015-5013

2. The ESPAD Group. ESPAD Report 2015, Results from the European School Survey Proyect on Alcohol and Other Drugs. Luxembourg: European Monitoring Centre for Drugs and Drug Addiction (2015).

3. Comisión Interamericana para el Control del Abuso de Drogas. Informe sobre Uso de drogas en las Américas. Washington, DC: Observatorio Interamericano de Drogas (2015).

4. Hawkins JD, Catalano RF, Miller JY. Risk and protective factors for alcohol and other drug problems in adolescence and early adulthood: implications for substance abuse prevention. Psychol Bull (1992) 112(1):64-105. doi:10.1037/0033-2909.112.1.64

5. Carvalho M, De Matos MG; Social Adventure Project Team. Psychosocial determinants of mental health and risk behaviours in adolescents. Glob J Health Sci (2014) 6(4):22-35. doi:10.5539/gjhs.v6n4p22

6. Brooks FM, Magnusson J, Spencer N, Morgan A. Adolescent multiple risk behaviour: an asset approach to the role of family, school and community. J Public Health (2012) 34(Suppl 1):i48-56. doi:10.1093/pubmed/ fds001

7. Goodenow C. The psychological sense of school membership among adolescents-scale development and educational correlates. Psychol Sch (1993) 30(1):79-90. doi:10.1002/1520-6807(199301)30:1<79::AID-PITS2310300113 $>3.0 . \mathrm{CO} ; 2-\mathrm{X}$

8. Boccanfuso C. "This Makes Me Who I Am": The Meaning and Significance of School Membership for Ninth Grade Students Transitioning to High School. College Park, MD: University of Maryland (2009).

9. Freeman TM, Anderman LH, Jensen JM. Sense of belonging in college freshmen at the classroom and campus levels. J Exp Educ (2007) 75(3):203-20. doi:10.3200/JEXE.75.3.203-220

10. Osterman KF. Students' need for belonging in the school community. Rev Educ Res (2000) 70(3):323-67. doi:10.3102/00346543070003323

11. Goodenow C. Classroom belonging among early adolescent students: relationships to motivation and achievement. J Early Adolesc (1993) 13(1):21-43. doi:10.1177/0272431693013001002

12. Bond L, Butler H, Thomas L, Carlin J, Glover S, Bowes G, et al. Social and school connectedness in early secondary school as predictors of late teenage substance use, mental health, and academic outcomes. J Adolesc Health (2007) 40(4):357.e9-18. doi:10.1016/j.jadohealth.2006.10.013

13. Resnick MD, Bearman PS, Blum RW, Bauman KE, Harris KM, Jones J, et al. Protecting adolescents from harm. Findings from the National Longitudinal Study on Adolescent Health. JAMA (1997) 278(10):823-32. doi:10.1001/ jama.1997.03550100049038

14. McNeely C, Falci C. School connectedness and the transition into and out of health-risk behavior among adolescents: a comparison of social belonging and teacher support. JSch Health (2004) 74(7):284-92. doi:10.1111/ j.1746-1561.2004.tb08285.x

15. McNeely CA, Nonnemaker JM, Blum RW. Promoting school connectedness: evidence from the National Longitudinal Study of Adolescent Health. J Sch Health (2002) 72(4):138-46. doi:10.1111/j.1746-1561.2002.tb06533.x

16. Henry KL, Swaim RC, Slater MD. Intraindividual variability of school bonding and adolescents' beliefs about the effect of substance use on future aspirations. Prev Sci (2005) 6(2):101-12. doi:10.1007/s11121-005-3409-0

17. Kim MJ, Fleming CB, Catalano RF. Individual and social influences on progression to daily smoking during adolescence. Pediatrics (2009) 124(3):895-902. doi:10.1542/peds.2008-2015

18. Napoli M, Marsiglia FF, Kulis S. Sense of belonging in school as a protective factor against drug abuse among Native American Urban Adolescents. J Soc Work Pract Addict (2003) 3(2):25-41. doi:10.1300/J160v03n02_03
Fellowship in the London School of Hygiene and Tropical Medicine, supported by a scholarship awarded from the National Commission for Scientific and Technological Research (CONICYT) and a scholarship from Universidad de los Andes (Chile).

19. Vaughan EL, Kratz L, d'Argent J. Academics and substance use among Latino adolescents: results from a national study. J Ethn Subst Abuse (2011) 10(2):147-61. doi:10.1080/15332640.2011.573315

20. Fletcher A, Bonell C, Sorhaindo A, Strange V. How might schools influence young people's drug use? Development of theory from qualitative case-study research. J Adolesc Health (2009) 45(2):126-32. doi:10.1016/ j.jadohealth.2008.12.021

21. Araya R, Montgomery AA, Fritsch R, Gunnell D, Stallard P, Noble S, et al. School-based intervention to improve the mental health of low-income, secondary school students in Santiago, Chile (YPSA): study protocol for a randomized controlled trial. Trials (2011) 12:49. doi:10.1186/1745-6215-12-49

22. Araya R, Fritsch R, Spears M, Rojas G, Martinez V, Barroilhet S, et al. School intervention to improve mental health of students in Santiago, Chile: a randomized clinical trial. JAMA Pediatr (2013) 167(11):1004-10. doi:10.1001/jamapediatrics.2013.2361

23. United Nations Office on Drugs and Crime. GAP Toolkit Module 3: Conducting School Surveys on Drug Abuse. Austria: United Nations (2003).

24. Servicio Nacional para la Prevención y Rehabilitación del consumo de drogas y alcohol (SENDA). Décimo Estudio Nacional de Drogas en Población Escolar 8 básico a 4 medio. Chile: Ministerio de Interior de Chile (2013).

25. Stallard P, Spears M, Montgomery AA, Phillips R, Sayal K. Self-harm in young adolescents (12-16 years): onset and short-term continuation in a community sample. BMC Psychiatry (2013) 13:328. doi:10.1186/1471-244X-13-328

26. Gaete J, Martinez V, Fritsch R, Rojas G, Montgomery AA, Araya R. Indicated school-based intervention to improve depressive symptoms among at risk Chilean adolescents: a randomized controlled trial. BMC Psychiatry (2016) 16:276. doi:10.1186/s12888-016-0985-4

27. Melipillán Araneda R, Cova Solar F, Rincón González P, Valdivia Peralta M. Propiedades Psicométricas del Inventario de Depresión de Beck-II en Adolescentes Chilenos. Terapia Psicol (2008) 26:59-69. doi:10.4067/S071848082008000100005

28. Araya R, Montero-Marin J, Barroilhet S, Fritsch R, Gaete J, Montgomery A. Detecting depression among adolescents in Santiago, Chile: sex differences. BMC Psychiatry (2013) 13:122. doi:10.1186/1471-244X-13-122

29. Stallard P, Phillips R, Montgomery AA, Spears M, Anderson R, Taylor J, et al. A cluster randomised controlled trial to determine the clinical effectiveness and cost-effectiveness of classroom-based cognitive-behavioural therapy (CBT) in reducing symptoms of depression in high-risk adolescents. Health Technol Assess (2013) 17(47):vii-xvii, 1-109. doi:10.3310/hta17470

30. Mackinnon DP, Dwyer JH. Estimating mediated effects in prevention studies. Eval Rev (1993) 17(2):144-58. doi:10.1177/0193841X9301700202

31. Simons-Morton B, Pickett W, Boyce W, ter Bogt TF, Vollebergh W. Crossnational comparison of adolescent drinking and cannabis use in the United States, Canada, and the Netherlands. Int J Drug Policy (2010) 21(1):64-9. doi:10.1016/j.drugpo.2009.02.003

32. White J, Walton D, Walker N. Exploring comorbid use of marijuana, tobacco, and alcohol among 14 to 15-year-olds: findings from a national survey on adolescent substance use. BMC Public Health (2015) 15:233. doi:10.1186/ s12889-015-1585-9

33. Miech RA, Johnston LD, O’Malley PM, Bachman JG, Schulenberg JE. Monitoring the Future National Survey Results on Drug Use, 1975-2014: Volume I, Secondary School Students. Ann Arbor: Institute for Social Research, The University of Michiga (2015).

34. Agalioti-Sgompou V, Chistie S, Fiorini P, Hawkins V, Hinchliffe S, Lepps H, et al. Smoking, Drinking and Drug Use Among Young People in England in 2014. Health and Social Care Information Centre (2015).

35. Needham BL, Crosnoe R, Muller C. Academic failure in secondary school: the inter-related role of health problems and educational context. Soc Probl (2004) 51(4):569-86. doi:10.1525/sp.2004.51.4.569

36. Clark DB, Pollock N, Bukstein OG, Mezzich AC, Bromberger JT, Donovan JE. Gender and comorbid psychopathology in adolescents with alcohol 
dependence. J Am Acad Child Adolesc Psychiatry (1997) 36(9):1195-203. doi:10.1097/00004583-199709000-00011

37. Hettema JM, Prescott CA, Kendler KS. The effects of anxiety, substance use and conduct disorders on risk of major depressive disorder. Psychol Med (2003) 33(8):1423-32. doi:10.1017/S0033291703008365

38. Swendsen JD, Merikangas KR, Canino GJ, Kessler RC, Rubio-Stipec M, Angst J. The comorbidity of alcoholism with anxiety and depressive disorders in four geographic communities. Compr Psychiatry (1998) 39(4):176-84. doi:10.1016/S0010-440X(98)90058-X

39. Marmorstein NR. Longitudinal associations between alcohol problems and depressive symptoms: early adolescence through early adulthood. Alcohol Clin Exp Res (2009) 33(1):49-59. doi:10.1111/j.1530-0277.2008.00810.x

40. Abraham HD, Fava M. Order of onset of substance abuse and depression in a sample of depressed outpatients. Compr Psychiatry (1999) 40(1):44-50. doi:10.1016/S0010-440X(99)90076-7

41. Diego MA, Field TM, Sanders CE. Academic performance, popularity, and depression predict adolescent substance use. Adolescence (2003) 38(149):35-42.

42. Bovasso GB. Cannabis abuse as a risk factor for depressive symptoms. Am J Psychiatry (2001) 158(12):2033-7. doi:10.1176/appi.ajp.158.12.2033

43. Daley SE, Hammen C, Rao U. Predictors of first onset and recurrence of major depression in young women during the 5 years following high school graduation. J Abnorm Psychol (2000) 109(3):525-33. doi:10.1037/0021-843X.109.3.525

44. Rao U, Daley SE, Hammen C. Relationship between depression and substance use disorders in adolescent women during the transition to adulthood. J Am Acad Child Adolesc Psychiatry (2000) 39(2):215-22. doi:10.1097/ 00004583-200002000-00022

45. Fletcher A, Bonell C, Hargreaves J. School effects on young people's drug use: a systematic review of intervention and observational studies. J Adolesc Health (2008) 42(3):209-20. doi:10.1016/j.jadohealth.2007.09.020

46. Perra O, Fletcher A, Bonell C, Higgins K, McCrystal P. School-related predictors of smoking, drinking and drug use: evidence from the Belfast Youth Development study. J Adolesc (2012) 35(2):315-24. doi:10.1016/ j.adolescence.2011.08.009
47. Gaete J, Montgomery A, Araya R. The association between school bonding and smoking amongst Chilean adolescents. Subst Abus (2015) 36(4):515-23. doi:10.1080/08897077.2014.991862

48. Drolet M, Arcand I, Ducharme D, Leblanc R. The sense of school belonging and implementation of a prevention program: toward healthier interpersonal relationships among early adolescents. Child Adolesc Social Work J (2013) 30:535-51. doi:10.1007/s10560-013-0305-5

49. Eisen M, Zellman GL, Murray DM. Evaluating the lions-quest "skills for adolescence" drug education program. Second-year behavior outcomes. Addict Behav (2003) 28(5):883-97. doi:10.1016/S0306-4603(01)00292-1

50. Jamal F, Fletcher A, Harden A, Wells H, Thomas J, Bonell C. The school environment and student health: a systematic review and meta-ethnography of qualitative research. BMC Public Health (2013) 13:798. doi:10.1186/14712458-13-798

51. Hibell B, Guttormsson U, Ahlstrom S, Balakireva O, Bjarnason T, Kokkevi A, et al. The 2011 ESPAD Report, Substance Use among Students in 36 European Countries. Sweden: Monitoring Centre for Drugs and Drug Addiction (EMCDDA) (2012).

52. Johnston LD, O'Malley PM, Miech RA, Bachman JG, Schulenberg JE. Monitoring the Future National Survey Results on Drug Use, 1975-2015: Overview, Key Findings on Adolescent Drug Use. Ann Arbor: Institute for Social Research, The University of Michigan (2016).

Conflict of Interest Statement: The authors declare that the research was conducted in the absence of any commercial or financial relationships that could be construed as a potential conflict of interest.

Copyright $(2018$ Gaete, Rojas, Fritsch and Araya. This is an open-access article distributed under the terms of the Creative Commons Attribution License (CC BY). The use, distribution or reproduction in other forums is permitted, provided the original author(s) and the copyright owner are credited and that the original publication in this journal is cited, in accordance with accepted academic practice. No use, distribution or reproduction is permitted which does not comply with these terms. 